\title{
$000-30.72-25$
}

\section{Hadronic Form Factors in Asymptotically Free Field Theories ${ }^{\dagger}$}

\section{David J. Gross* and S.B. Treiman \\ Joseph Henry Laboratories \\ of Physics \\ Princeton University \\ Princeton, New Jersey \\ 08540}

\section{NOTICE}

account of work This report was prepared as an accounent. Neither sponsored by the United States Sovtes Atomic Energy the United States nor the United Stales An any of Commission, nor any of their employees, employees, Commission, subcontractors, or their employes, their conr warranty, express or implied, or assumes any makes any warre responsibility for the accuracy, com legal liability or respons any information, apparatus,
pleteness or usefulness of any

product or process privately owned rights.

\begin{abstract}
The breakdown of Bjorken scaling in asymptotically free gauge theories of the strong interactions is explored for its implications on the large $\mathrm{q}^{2}$ behavior of nucleon form factors. Duality arguments of Bloom and Gilman suggest a connection betwaen the form factors and the threshold properties of the deep inelastic structure functions. The latter are addressed directly in an analysis of asymptotically free theories; and through the duality connection we are then led to statements about the form factors. For very large $\mathrm{q}^{2}$ the form factors are predicted to fall faster than any inverse power of $q^{2}$. For the more modest range of $q^{2}$ reached in existing experiments the agreement with data is fairly good, though this may well be fortuitous. Extrapolations beyond this range are presented.
\end{abstract}

† Research supported in part by the U.S. Atomic Energy Commission under Contract No. AT $(11-1)-3072$

* Alfred P. Sloan Foundation Fellow. 


\section{DISCLAIMER}

This report was prepared as an account of work sponsored by an agency of the United States Government. Neither the United States Government nor any agency Thereof, nor any of their employees, makes any warranty, express or implied, or assumes any legal liability or responsibility for the accuracy, completeness, or usefulness of any information, apparatus, product, or process disclosed, or represents that its use would not infringe privately owned rights. Reference herein to any specific commercial product, process, or service by trade name, trademark, manufacturer, or otherwise does not necessarily constitute or imply its endorsement, recommendation, or favoring by the United States Government or any agency thereof. The views and opinions of authors expressed herein do not necessarily state or reflect those of the United States Government or any agency thereof. 


\section{DISCLAIMER}

Portions of this document may be illegible in electronic image products. Images are produced from the best available original document. 
In a recent paper ${ }^{1}$ by one of us (D.J.G.) the breakdown of Bjorken scaling was investigated in the context of asymptotically free gauge theories of the strong interactions. ${ }^{2,3}$ An asymptotic extrapolation formula was obtained that expresses the deep inelastic structure function $F_{2}\left(\omega, q^{2}\right)$ at one value of $q^{2}$ in terms of the function $F_{2}\left(w, q^{\prime 2}\right)$ at another value, $q^{\prime 2}$, of the momentum transfer variable - provided both $\mathrm{q}^{2}$ and $\mathrm{q}^{\prime 2}$ are large enough to be in the "asymptotic" region. It was shown that deviations from scaling are expected to be especially large in the vicinity of threshold $\left(\omega \equiv\left(2 v+m^{2}\right) / q^{2} \approx 1\right) .^{4}$ Here we wish to explore the consequences which this breakdown of scaling would suggest for a related topic: the $q^{2}$ dependence of elastic and transition form factors of nucleons.

First, let us recall some results from Ref. 1 . One starts with the asymptotic relation ${ }^{2,5}$ for the moments of the structure function $\nu W_{2}=\mathrm{mF}_{2}$ :

$$
\int_{1}^{\infty} d w F_{2}\left(w, q^{2}\right) w^{-N-2} \underset{q^{2} \rightarrow \infty}{\longrightarrow} C_{N}\left(\ln q^{2} / u^{2}\right)^{-A_{N}},
$$

where the $C_{N}$ are unspecified constants and $\mu$ is an unspecified scale parameter. The exponents $A_{N}$ are related to the calculable 2,5 anomalous dimensions of the dominant operators of spin $\mathrm{N}+2$ in the Wilson expansion for a product of currents. With

$$
t=l_{22} \frac{q^{2}}{a^{2}}, \quad t^{\prime}=l_{11} \frac{g^{\prime 2}}{a^{2}}, t>t^{\prime},
$$

one now derives the asymptotic extrapolation formula 


$$
F_{2}(w, t)=\int_{1}^{\omega^{\prime}} \frac{d w^{\prime}}{\omega^{\prime}} F_{2}\left(\frac{\omega}{\omega^{\prime}}, t^{\prime}\right) T\left(\omega^{\prime}, \frac{t}{t^{\prime}}\right)
$$

where $(\sigma>0)$

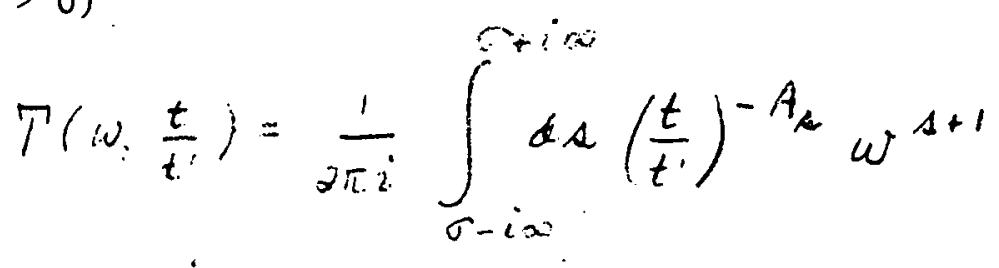

Equation (2) is supposed to become exact when $t$ and $t^{\prime}$ are in the asymptotic region, i.e., where the effective "coupling constant $\bar{g}\left(t^{\prime}\right)$ is sufficiently'small compared to unity.

As written, Eq. (1) represents the situation that would obtain if there were a single operator which controls the asymptotic behavior of the $\mathrm{N}^{\text {th }}$ moment. In fact there are several such operators ${ }^{6}$ (singlets of normal and abnormal parity, as well as a non-singlet). In general, for any given $N$, these have different anomalous dimensions, so the right side of Eq. (1) shuuld be replaced by a corresponding sum of contributions from the several operators. However, we are interested here in the behavior of the structure function $F_{2}$ near the threshold, at $\omega=1$. This is governed by the moments of large $\mathrm{N}$; and it turns out that the anomalous dimensions of the various operators all have the same limiting behavior for large $N$. Therefore, the single term on the right side of $\mathrm{Eq}$. (1) represents the net effect at large $\mathrm{N}$. In this limit one has

$$
A_{N} \longrightarrow[\operatorname{lin}(N+2)-0.69]
$$

where $G$ is determined by the structure of the strong gauge group and by the quark representation under the group. If the strong gauge group is $S U(3)^{\prime}$, and if the theory contains three quark triplets, then one finds 
4 .

that $G=2 / 27$. This is the numerical value that we shall adopt for later numerical purposes.

Suppose that the reference structure function, at $t^{\prime}$, has the following behavior near threshold:

$$
F_{2}\left(\omega, t^{\prime}\right) \sim(\omega-1)^{d}, \quad \omega \rightarrow 1 .
$$

Then the structure function at $t>t^{\prime}$ has itself the rather simple threshold behavior given by

$$
F_{2}(\omega, t) / F_{2}\left(\omega, t^{\prime}\right) \underset{\omega \rightarrow 1}{\rightarrow}\left(\frac{t}{t^{\prime}}\right)^{.69 G} \frac{\Gamma(d+1)}{\Gamma(a+1+P)}(\ln \omega)^{p}
$$

where

$$
P=4 G \ln \frac{t}{t^{\prime}}
$$

The threshold property expressed by Eq. (5) seems in fact to obtain (with $\mathrm{d} \approx 3$ ) in the $\mathrm{q}^{\prime 2}$ region relevant to the SLAC-MIT experiments.

Accepting this, we now wish to consider the implications of Eq. (6) for the question of nucleon electromagnetic form factors: Consider the contributton to the structure function $F_{2}$ coming from some resonance of mass $M_{r}$. For simplicity we take the width to be infinitely narrow. This contribution is

$$
F_{2}^{r}=\nu G^{2}\left(q^{2}\right) \delta\left(\nu-\nu_{r}\right)
$$


where $v_{r}=\frac{1}{2}\left(M_{r}^{2}-m^{2}+q^{2}\right)$. This equation defines the electromagnetic transition form factor connecting the nucleon and the resonance. If the "resonance" in question is the nucleon itself $\left(M_{r}^{2}=m^{2}\right)$, then we are dealing with the diagonal electric and magnetic form factors and

$$
G^{2}=\left[G_{E}^{2}+\left(q^{2} / 4 m^{2}\right) G_{M}^{2}\right] /\left[1+\left(q^{2} / 4 m^{2}\right)\right]
$$

Accepting that the second term dominates the first term in the numerator of Eq. (9). at large $\mathrm{q}^{2}$, we have

$$
G^{2} \underset{q^{2} \rightarrow \infty}{\rightarrow} G_{M}^{2}\left(1+O\left(1 / q^{2}\right)\right)
$$

with $M_{0}>M_{r}$, it now follows from positivity that

$$
\int_{1+m^{2} / q^{2}}^{1+m_{0}^{2} / q^{2}} d w F_{2}\left(q^{2}, \omega\right)>G^{2}\left(q^{2}\right) .
$$

As $\mathrm{q}^{2} \rightarrow \infty$, the integration is restricted to the threshold region of $\omega$, and we may therefore adopt Eq. (6) for $F_{2}\left(q^{2}, w\right)$. In this way we find

$$
G^{2}\left(q^{2}\right)<\text { constant }\left(\frac{t}{t^{\prime}}\right)^{.69 G} \frac{\Gamma(d+2)}{\Gamma(d+2+P)}\left(\frac{S_{0}}{q^{2}}\right)^{d+1+P},
$$

where $s_{0}=M_{0}^{2}-m^{2}$. Notice that the parameters $d$ and $q^{\prime 2}$ depend on the choice of reference structure function, according to Eq.(5). As Eq. (6) shows $d$ increases with $q^{12}$. 
In deriving Eq. (10) we have assumed that the limit $\mathrm{q}^{2} \rightarrow \infty$ is uniform in $\omega$, even in the vicinity of threshold, $\omega \approx 1+0\left(\frac{1}{\mathrm{q}} 2\right)$. This could be false if the corrections to Eq. (1), which are of order $\left(\ln \frac{q^{2}}{\mu^{2}}\right)^{-1}$, appear with coefficients that increase sufficiently rapidly for large $N$ (faster than $\ln N$ ). In the present paper we assume that the corrections to Eq. (1) do not have such rapidly growing coefficlents; or if they do, that this nevertheless does not destroy the uniformity of the large $q^{2}$ limit near threshold. ${ }^{7}$ Accepting this, we see for asymptotically free gauge theories that the nuclear elastic and transition form factors must fall faster than any inverse power of $q^{2}$ as $q^{2} \rightarrow \infty$; indeed, faster than $\left(q^{2}\right)^{-2 G l n \ell n q^{2}}$.

Returning to the arguments leading to Eq.(11), we observe that a more predictive result can be obtained if we invoke the notion of local duality, as advocated for electroproduction by Bloom and Gilman. 8 In this context duality means that the resonant, or the elastic, contribution is some constant fraction, independent of $\mathrm{q}^{2}$, of the structure function, even as $q^{2} \rightarrow \infty$. Consider for example the nucleon contribution itself. Duality implies that the two sides of Eq.(10) are proportional to each other at all $q^{2}$, where $M_{0}{ }^{2}$ should be chosen to render the proportionality constant as close to unity as possible. From the SLAC-MIT data, Bloom and Gilman 8 find that $s_{0} \approx(1.23 \mathrm{Gev})^{2}$ is the best choice. Using this version of Eq. (10), both at $\mathrm{q}^{2}$ and $\mathrm{q}^{\prime 2}$ one finds, for the nucleon magnetic form factor

$$
\frac{G_{M}^{2}\left(q^{2}\right)}{G_{M}^{2}\left(q^{\prime 2}\right)} \approx\left(\frac{\ln q^{2} / \mu^{2}}{\ln q^{\prime 2} / \mu^{2}}\right)^{.69 G} \frac{\Gamma(\alpha+1)}{\Gamma(\alpha+2+p)}\left(\frac{s_{0}}{q^{2}}\right)^{p}\left(\frac{q^{\prime 2}}{q^{2}}\right)^{\alpha+1}
$$


provided both $q^{\prime}$ and $q$ are large enough. The same formula holds for transition form factors to resonances. As noted earlier, all the revelant twist-two operators have the same anomalous dimensions for large $\mathrm{N}$, irrespective of isospin and aher quantum numbers. It follows that the asymptotic extrapolation formula of Eq. (12) holds separately for the nucleon isovector and isoscalar magnetic form factors and similarly for the corresponding axial vector form factors.

The analysis given above can be repeated for the longitudinal structure function $F_{L}\left(q^{2}, \omega\right)$, which receives contributions from the nucleon electric form factor $G_{E}\left(q^{2}\right)$ : In asymptotically free theories of the type discussed in Ref. 2 the moments of $F_{L}$ are asymptotically smaller by one power of $\log \mathrm{q}^{2} / \mathrm{\mu}^{2}$ than the corresponding moments of $\mathrm{F}_{2}$. Moreover, the $\mathrm{N}$ dependence of the ratios of the coefficients can be explicitly computed. ${ }^{9}$ Using the results thus obtained, and combining this with an analysis of the sort described in Ref. 1 , one finds near threshold $(\omega \rightarrow 1)$ that $F_{L} / F_{2} \rightarrow(1-\omega)\left(\operatorname{lnq}^{2} / \mu^{2}\right)^{-1}$. On the basis of the duality qrguments employed here one now finds that $G_{E}{ }^{2} / G_{M}{ }^{2} \rightarrow\left(\operatorname{lnq}{ }^{2} / \mu^{2}\right)^{-1}$ as $q^{2} \rightarrow 0$. Thus the "scaling" of electric and magnetic form factors (to within logarithms) emerges as a prediction of asymptotically free theories based on spin one-half constituents.

Beyond the major uniformity and duality assumptions that have gone into Eq. (12), there are several practical delicacies that arise in any attempt to use this equation for the regions of $q^{2}$ that are presently accessible to experiment. We have to start at some reference $\mathrm{q}^{\prime 2}$ where the structure function $F_{2}\left(\omega, q^{\prime 2}\right)$ is known in the vicinity of $\omega=1$; 'and we have to suppose that $\mathrm{q}^{\prime 2}$ is large enough to be regarded as asymptotic. It is an open question whether present experiments have penetrated this asymptotic 
region. Certainly the present evidence is too sparse to determine, for Eq. (5), whether the exponent $d$ is varying with $q^{2}$ over the limited range covered. To be sure, Eqns.(6) and (7) suggest that $d$ should not vary rapidly with $\mathrm{q}^{2}$ and we shall accept the experimental ${ }^{10}$ result, $d=3$, as applying to any $\mathrm{q}^{\prime 2}$ in the region of a few (Gev) ${ }^{2}$. From Eq. (12) we see, for any 1imited range in the vicinity of the reference $q^{\prime 2}$, that the form factor variation with $q^{2}$ comes chiefly from the factor $\left(q^{2}\right)^{-d-1}$ on the right-hand side of the equation. With $d=3$ this implies $G_{M} \sim\left(q^{2}\right)^{-2}$, which is the celebrated dipole behavior - in rough agreement with experiment up to $q^{2} \sim$ few (Gev) $)^{2}$. In this region actually, the behavior is more accurately represented by

$$
G_{M} \sim\left(1+q^{2} / q^{2}\right)^{-2},
$$

with $\mathrm{q}_{0}{ }^{2}=0.71(\mathrm{Gev})^{2}$. This departure from Eq.(12), together with the other departure of order $\left(\mathrm{q}^{2}\right)^{-1}$ represented by the approximation in going from Eq. (9) to Eq. ( $\left.9^{\prime}\right)$, might well arise from higher order corrections to Eq. (5); i.e., from a next term of order $(\omega-1)^{d+1}$. Indeed the successful duality fits of Bloom and Gilman suggest that this is so. For practical purposes we incorporate these effects into Eq. (12) by replacing the factor $\left(q^{12} / q^{2}\right)^{d+1}$ with the factor $\left(\mathrm{q}^{\prime 2}+\mathrm{q}_{0}{ }^{2}\right)^{\mathrm{d}+1} /\left(\mathrm{q}^{2}+\mathrm{q}_{0}{ }^{2}\right)^{\mathrm{d}+1}, \mathrm{~d}=3$. For $\mathrm{q}^{2}>\mathrm{q}^{\prime 2}>>\mathrm{q}_{0}{ }^{2}$, this amounts to a correction of order $\left(q^{2}\right)^{-1}$.

Apart from the choice of a reference $q^{\prime 2}$, Eq. (12) contains the parameters $G, \mu$, and $s_{0}$. The first is specified by the gauge group and its quark content. For present purposes, as mentioned earlier, we shall take $G=2 / 27$, corresponding to $\mathrm{SU}(3)^{\prime}$ and three quark triplets. As for the parameter $s_{0}$, this does not enter sensivively into Eq.(12), since 
the function $P$ vanishes at $q^{2}=q^{\prime 2}$ and increases only very slowly as $q^{2}$ increases beyond $q^{\prime 2}$. We adopt for $s_{0}$ the Bloom-Gilman value noted previously. Finally, there is the choice to be made of the scale parameter $\mu$. If the reference $q^{\prime 2}$ that we choose is supposed to be sufficiently asymptotic, then we must certainly suppose that $\mu^{2} \ll q^{\prime 2}$. We have considered several cases to illustrate how the form factor predictions vary with the choices of $\mathrm{q}^{12}$ and $\mu^{2}$. The solid curves in Fig. 1 show the results, for $q^{12}=5$, with $\mu^{2}=0.5$ and 1.0 [the units are $(\mathrm{Gev})^{2}$ ]. The solid curves in Fig. 2 correspond to $q^{12}=10, \mu^{2}=0.5$ and 2.0 . Both figures also display the experimental points. ${ }^{11}$

of course if our whole speculative analysis is right, if we know the correct scale parameter $\mu$, and if we know the correct exponent parameter $d\left(q^{\prime 2}\right)$, then the results should not depend on the choice of $q^{\prime 2}$ - if it is sufficiently asymptotic. This is a large collection of "ifs". In fact, as the curves of Figs. 1 and 2 show, the two choices $q^{\prime 2}=5$ and $q^{\prime 2}=10$ indeed do not produce markedly different results, Similarly the sensitivity to the scale parameter $\mu$ seems to be only moderate. That the various curves show some resemblance to the existing data is another matter. Especially at the larger values of $\mathrm{q}^{2}$ the error flags are not negligible, and anyhow, the rough agreement with the curves may well be fortuitous.

What is qualitatively significant is the experimental indication of a fall off below the dipole formula at large $q^{2} \cdot 12$ If Bjorken scaling holds exactly, then the Bloom-Gilman analysis suggests that $G_{M}$ should fall exactly like $\left(q^{2}\right)^{-2}$. The more rapid fall off observed to set in around $q^{2} \approx 5$ would then imply either a fallure of the duality arguments, or a breakdown of exact Bjorken scaling. It is this latter interpretation that we have adopted here, in the context of asymptotically free gauge theories. The 
10 .

interesting circumstance arises that one can get at a possible breakdown of exact scaling indirectly, through the elastic form factor; as well as directly, through the deep inelastic structure functions. The breakdown effects are magnified for the form factor, as one sees by comparing the $q^{2}$ dependence of Eqns. (6) and (12). It is clearly worthwhile to pursue both approaches, out to the largest possible values of $q^{2}$. 


\section{References and Footnotes}

1. D.J. Gross, "How to Test Scaling in Asymptotically Free Theories", Rockefeller preprint (1974).

2. D.J. Gross and F. Wilczek, Phys. Rev. Letters 30, 1343 (1973); Phys. Rev.

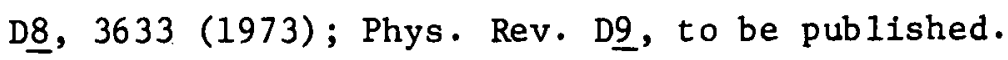

3. H.D. Politzer, Phys. Rev. Letters 30, 1346 (1973).

4. Since we shall invoke duality arguments in this paper, it is convenient to use the Bloom-Gilman scaling variable instead of the more conventional $\omega=2 v / q^{2}$.

5. H. Georgi and H.D. Politzer, Phys. Rev. D9, 416 (1974).

6. The anomalous dimensions of the singlet, normal parity operator and of the non-singlet operators were calculated in Refs. 2 and 5 . The anomalous dimensions of the abnormal parity singlet operators, which contribute to $W_{3}$, were calculated by one of us (D.J.G., unpublished); again, for large $\mathrm{N}$, the results are as in $\mathrm{Eq} \cdot(4)$.

7. There are some indications that the corrections to Eq. (1) may indeed behave as $\bar{g}^{2} \ell^{2} \mathrm{~N}$ (relative to unity for the leading term). For Eq.(6) this would produce a relative correction of order $(\ln \ln w)^{2} / \ln \left(q^{2} / \mu^{2}\right)-$ which behaves like $\log q^{2} / \mu^{2}$ for $\omega=1+0\left(1 / q^{2}\right)$. If this situation really persists, one would then have to imagine summing over the corrections to Eq. (1) of all orders in $\vec{g}^{2}$, in order to test the validity of our basic assumption. This will be a difficult task. For the present we stick to our assumptions.

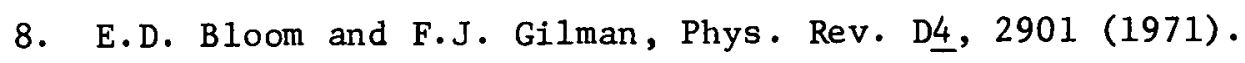

9. M. Calvo, private communication (to be published).

10. G. Miller, et.al., Phys: Rev. Dㅌ, 528 (1972). 


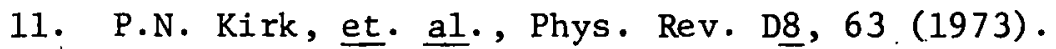

12. As has already been noted, with exact Bjorken scaling the duality argument implies that $G_{M}$ falls like $\left(q^{2}\right)^{-\frac{(d+1)}{2}}$. Given that $d=3$ in the SLAC - MIT region this leads to the simple dipole behavior; but nothing in the argument explains why $d=3$. However, the dipole behavior is sometimes thought to follow from the assumption that the nucleon is a bound state of three quarks; this is the picture embodied in the dimensional rules of S. Brodsky and G. Farrar, Phys. Rev. Letters 31,1153 (1973). - This picture has been shown to emerge in non-gauge theorles for which the dimensions are taken to be canonical (C.G. Callan and D.J. Gross, to be published). However, in asymptotically free gauge theories there appear to be logarithmic corrections. These have not so far been calculated. For consistency with the results obtained in the present paper, one has to suppose that these corrections sum up to give the modifications to the dipole formula which are embodied in Eq.(12). As seen, these modifications become very substantial indeed at large $\mathrm{q}^{2}$, even though compounded out of logarithms. 
Fi.gure Captions

1. The quantity $\left(1+\mathrm{q}^{2} / \mathrm{q}_{0}{ }^{2}\right) \mathrm{G}_{\mathrm{M}}{ }^{2}\left(\mathrm{q}^{2}\right) / \mathrm{G}_{\mathrm{M}}{ }^{2}(0), \mathrm{q}_{0}{ }^{2}=0.71(\mathrm{Gev})^{2}$, as a function of $\mathrm{q}^{2}$, for the choice of reference point $\mathrm{q}^{12}=5(\mathrm{Gev})^{2}$. The upper curve corresponds to scale parameter $\mu^{2}=0.5(\mathrm{Gev})^{2}$; the lower curve to $\mu^{2}=1.0(\mathrm{Gev})^{2}$. The dat a points are from Reference 11.

2. The quantity $\left(1+q^{2} / q_{0}{ }^{2}\right)^{2} G_{M}{ }^{2}\left(q^{2}\right) / G_{M}{ }^{2}(0), q_{0}{ }^{2}=0.71(\mathrm{Gev})^{2}$, as a function of $q^{2}$, for the choice of reference point $q^{\prime 2}=10(\mathrm{Gev})^{2}$. The upper curve corresponds to $\mu^{2}=0.5$ (Gev) ${ }^{2}$; the lower curve t.o $\mu^{2}=2.0(\mathrm{Gev})^{2}$. 


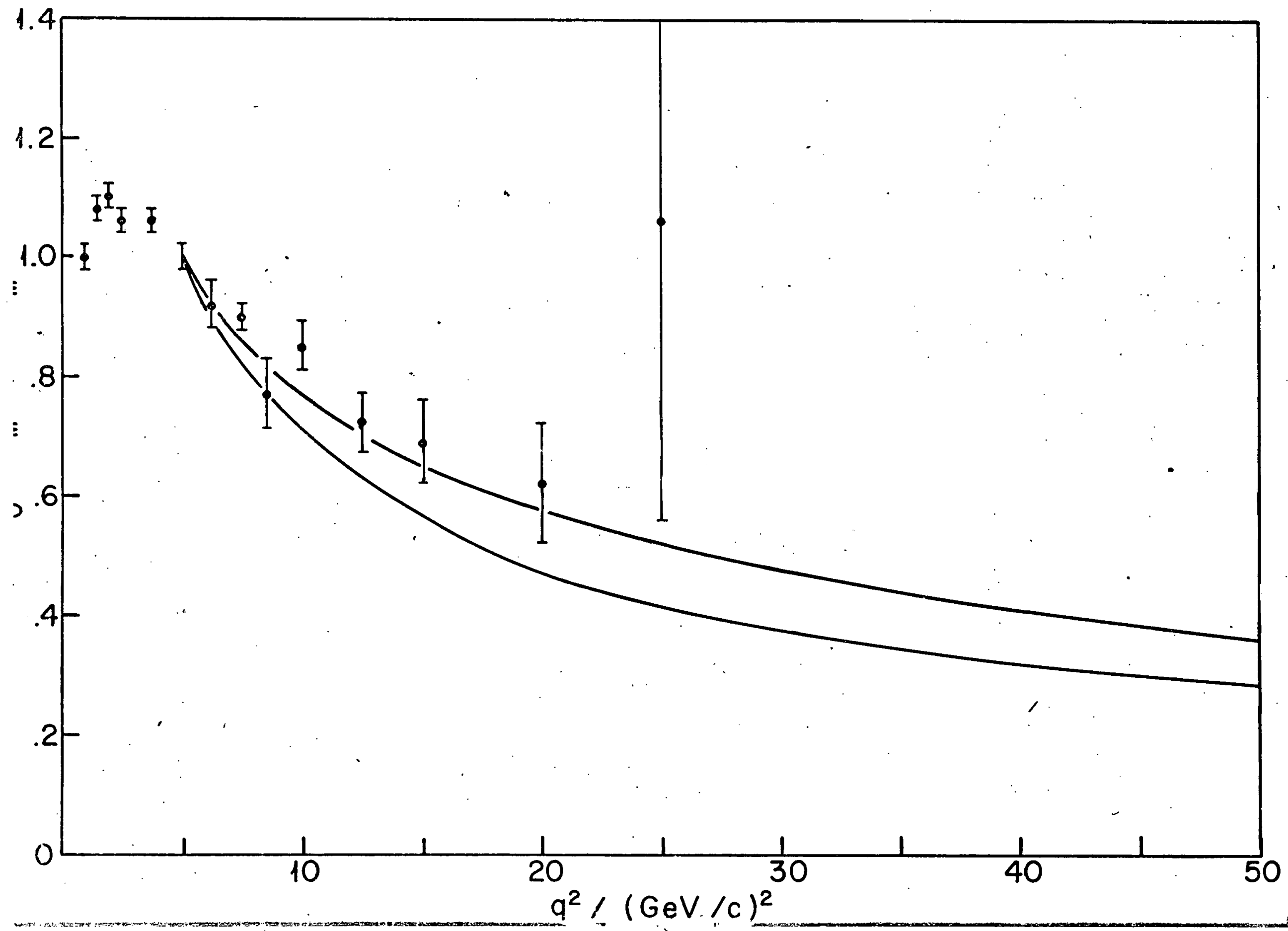




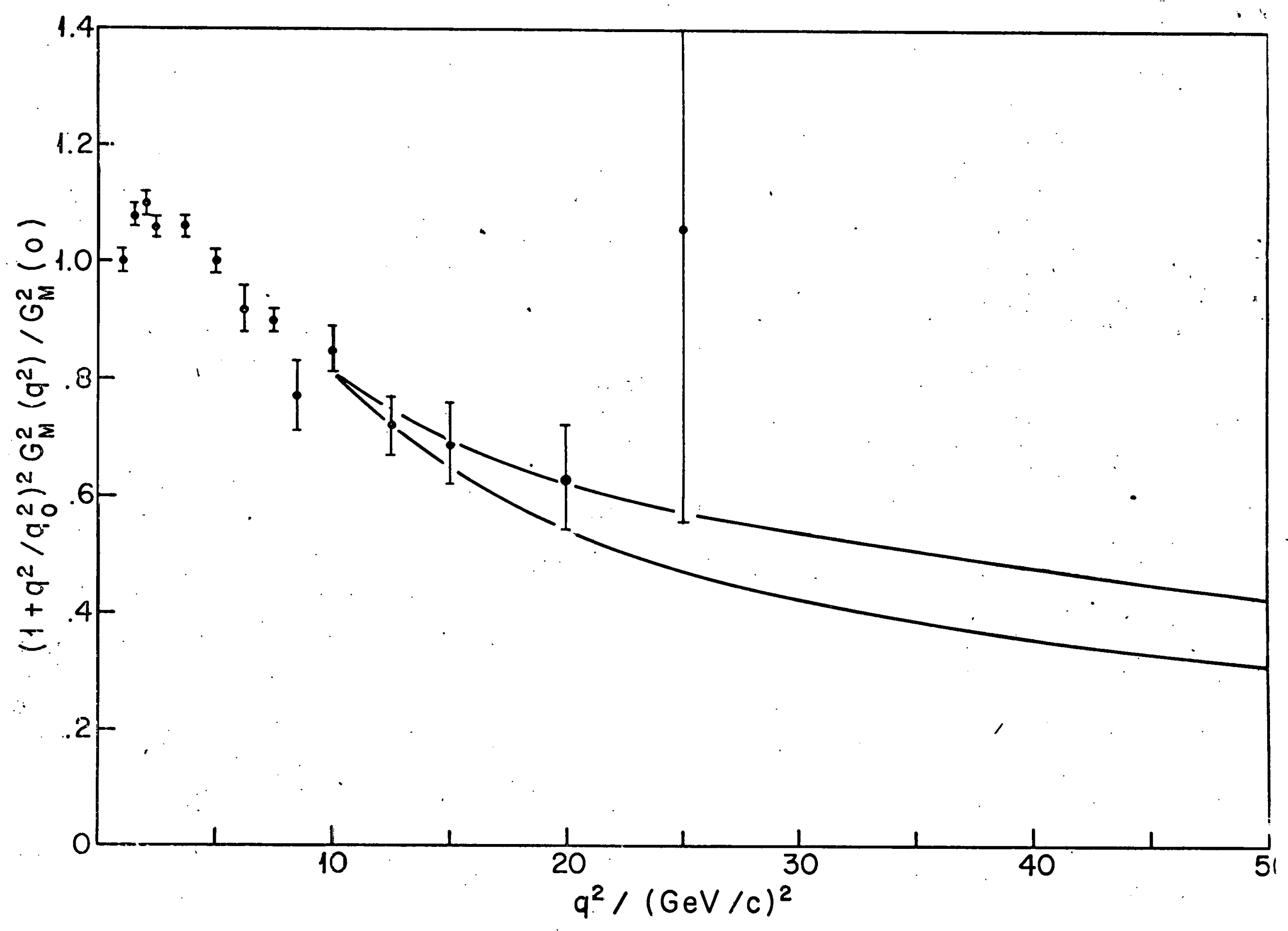

